\title{
The expression and potential mechanism of EGFR and EZH2 in breast cancer
}

\author{
Xiaoqi Tang ${ }^{1}$, Taosheng Zhou ${ }^{1}$, Jiayue Shen ${ }^{1}$, Ming Luo ${ }^{1}$, Huiming Yuan ${ }^{1}$, Denghua Pan ${ }^{2}$, Fu Li $^{1}$ \\ ${ }^{1}$ Department of Gastrointestinal and Gland Surgery, First Affiliated Hospital of Guangxi Medical University, Nanning, China; ${ }^{2}$ Department of \\ Ultrasonography, Second Affiliated Hospital of Guangxi Medical University, Nanning, China \\ Contributions: (I) Conception and design: X Tang, F Li; (II) Administrative support: F Li, D Pan; (III) Provision of study materials or patients: M Luo, \\ H Yuan; (IV) Collection and assembly of data: X Tang, T Zhou, J Shen; (V) Data analysis and interpretation: X Tang; (VI) Manuscript writing: All \\ authors; (VII) Final approval of manuscript: All authors. \\ Correspondence to: Prof. Fu Li. Department of Gastrointestinal and Gland Surgery, First Affiliated Hospital of Guangxi Medical University, 6 \\ Shuangyong Road, Nanning 530021, China. Email: 266321@163.com; Dr. Denghua Pan. Department of Ultrasonography, Second Affiliated \\ Hospital of Guangxi Medical University, 166 Daxuedong Road, Nanning 530007, China. Email: 1033307479@qq.com.
}

Background: The purpose of our research was to investigate the expression of epidermal growth factor
receptor $(E G F R)$ and zeste gene enhancer homolog $2(E Z H 2)$ in breast cancer, and to explore their potential
common pathways.
Methods: Western blot and quantitative real-time polymerase chain reaction (qRT-PCR) were used
to detect the protein and corresponding mRNA expression of EGFR and EZH2 in breast cancer tissues
and benign tissues. Then, the relationship between $E G F R$ and $E Z H 2$ along with the corresponding
clinicopathological parameters were also analyzed. Bioinformatics tools were applied to explore the possible
common pathways.

Results: The results showed that both EGFR and EZH2 protein and mRNA were highly expressed in breast cancer tissues, and there was a positive correlation between EGFR and EZH2. Moreover, we found that increased mRNA expression was correlated with lymph node metastasis and clinical stage $(\mathrm{P}<0.05)$. Furthermore, the enrichment results of co-expressed genes indicated that EGFR and EZH2 may work together in the FOXO signaling pathway, affecting the growth and metastasis of breast cancer cells.

Conclusions: The high expression of both EGFR and EZH2 mRNA in breast cancer was related to lymph node metastasis and clinical staging. The FOXO signaling pathway may be their common signaling pathway that affects tumor cell invasion and metastasis.

Keywords: Breast cancer; epidermal growth factor receptor $(E G F R)$; zeste gene enhancer homolog 2 (EZH2); infiltration and metastasis; FOXO signaling pathway

Submitted Jul 02, 2021. Accepted for publication Aug 17, 2021.

doi: $10.21037 / g s-21-505$

View this article at: https://dx.doi.org/10.21037/gs-21-505

\section{Introduction}

Breast cancer is one of the most common tumors in women worldwide, with an incidence rate of about $16 \%$ of all tumors, and its related mortality ranks first among female malignant tumors (1). In China, women who died from breast cancer alone in 2014 accounted for $7.82 \%$ of the total deaths from female-related cancers (2). In fact, the average age at Chinese women diagnosed with breast cancer is $45-55$ years old, which is lower than the average of women in western countries, because of the application of assisted reproductive technology, birth patterns, lifestyle, environmental factors and so on $(3,4)$. Epidermal growth factor receptor (EGFR) activation can induce phosphorylation of ERBB dimer partners, thereby 
affecting intracellular pathways such as RAS, RAF, MEK, ERK, PI3K, AKT, TOR, and Src kinase, and transcription factors such as STAT (5). At present, studies have proven that $E G F R$ plays an important role in the occurrence and development of bladder cancer, prostate cancer, and other malignant tumors (6-8). Breast cancer is also involved in the same procedure (9). Zeste gene enhancer homolog 2 (EZH2) is a member of the polycomb group (PcG) family. A study demonstrated that EZH2 can affect the stability of ribosomal DNA, thereby promoting the growth and metastasis of breast cancer cells (10). The inhibition of the catalytic activity of EZH2 can target the metastatic subtype of triple negative breast cancer (TNBC) (11). Although EGFR and EZH2 have the similar effects, there are few studies which focus on the effect of $E G F R$ and EZH2 co-expression on breast cancer. Additionally, whether EGFR and EZH2 have a common mechanism of action in the process of breast cancer is still unclear. This study analyzed the expression of EGFR and EZH2 at the protein and mRNA levels, along with their association with clinicopathological factors. The possible common signaling pathways were also investigated, as well as the mechanisms of invasion and metastasis of breast cancer. We present the following article in accordance with the REMARK reporting checklist (available at https://dx.doi. org/10.21037/gs-21-505).

\section{Methods}

\section{Patients}

A total of 120 cases of cancer tissues were collected from patients who were diagnosed with invasive breast cancer in the Department of Gastrointestinal and Gland Surgery of the First Affiliated Hospital of Guangxi Medical University from September 2017 to September 2018. The patients were aged from 29 to 74 years old, with an average age of $50.26 \pm 10.63$ years old. At the same time, 30 breast specimens that were obtained by Mammotome surgery and whose pathological results were benign lesions were collected. Patients were aged from 21 to 68 years old, with an average age of $41.41 \pm 11.31$ years old. All procedures performed in this study involving human participants were in accordance with the Declaration of Helsinki (as revised in 2013). The study was approved by institutional ethics board of First Affiliated Hospital of Guangxi Medical University [No. 2021(KY-E-038)] and informed consent was taken from all the patients.

\section{Western blot analysis}

The total protein was extracted using radioimmunoprecipitation (RIPA) protein lysate (Solarbio, Beijing, China). The protein concentration was determined by the BCA method. The protein samples were electrophoresed on a polyacrylamide gel and then transferred to a polyvinylidene fluoride (PVDF) membrane (Solarbio, Beijing, China). After blocking with $5 \%$ skim milk, the membrane was incubated with the primary antibody (Cell Signal Technology, Danvers, MA, USA) at $4{ }^{\circ} \mathrm{C}$ overnight. After washing with Tris buffered saline Tween (TBST), the membrane was incubated with the secondary antibody. The developer solution was added and allowed to soak for 2 minutes, then was placed in the analyzer (BIO-RAD, Hercules, CA, USA) for development.

\section{Quantitative real-time polymerase chain reaction (qRT-PCR)}

An RNA extraction kit (TAKARA, Dalian, China) was used to extract total RNA from tissues, then an ultraviolet spectrophotometer (Thermo Fisher Scientific, Waltham, MA, USA) was used to measure the RNA concentration. Subsequently, cDNA was synthesized according to the instructions of the reverse transcription kit (TAKARA). The reaction conditions of qRT-PCR were pre-denaturation at $95^{\circ} \mathrm{C}$ for $30 \mathrm{~s}$, denaturation at $95^{\circ} \mathrm{C}$ for $5 \mathrm{~s}$, and annealing at $60^{\circ} \mathrm{C}$ for $34 \mathrm{~s}$, for a total of 40 cycles. The $2^{-\Delta \Delta \mathrm{Ct}}$ method was used to calculate the relative expression level.

The primer sequences used in this experiment were as follows:

GAPDH: upstream primer: 5'-GCA CCG TCA AGG CTG AGA AC-3'; downstream primer: 5'-TGG TGA AGA CGC CAG TGG A-3'.

EZH2: upstream primer: 5'-AAA TCA GAG TAC ATG CGA CTG A-3'; downstream primer: 5'-GTA TCC TTC GCT GTT TCC ATT C-3'.

EGFR: upstream primer: 5'-ACC CAT ATG TAC CAT CGA TGT C-3'; downstream primer: 5'-GAA TTC GAT GAT CAA CTC ACG G-3'.

\section{Bioinformatics tool analysis}

Using the online database cBioPortal (12), two datasets (METABRIC, Nature 2012 \& Nat Commun 2016, 2,509 samples; TCGA, PanCancer Atlas, 1,084 samples) were used to select the first 400 genes co-expressed with $E G F R$ and $E Z H 2$. The Spearman correlation coefficient was set to 
Table 1 Comparison of the basic characteristics between the breast cancer group and the benign breast lesion group

\begin{tabular}{lcccc}
\hline Parameters & Benign & Breast cancer & $\mathrm{t}$ & $\mathrm{P}$ value \\
\hline Age & $41.41 \pm 11.31$ & $50.26 \pm 10.63$ & 3.971 & 0.000 \\
Height & $157.38 \pm 4.56$ & $156.22 \pm 5.78$ & 1.516 & 0.132 \\
Body weight & $53.44 \pm 7.06$ & $56.14 \pm 8.93$ & -1.009 & 0.315 \\
\hline
\end{tabular}

Table 2 Comparison of basic characteristics in different subtypes of the breast cancer group

\begin{tabular}{lcccccc}
\hline Parameters & LA & LB HER2 $(+)$ & HER2 $(+)$ & TNBC & F & P value \\
\hline Age & $48.10 \pm 10.57$ & $51.31 \pm 13.03$ & $52.96 \pm 8.96$ & $49.03 \pm 8.71$ & 1.310 & 0.275 \\
Height & $156.17 \pm 5.39$ & $156.73 \pm 7.31$ & $155.38 \pm 4.58$ & $155.93 \pm 6.09$ & 0.571 & 0.635 \\
Body weight & $56.74 \pm 8.27$ & $55.77 \pm 7.31$ & $56.12 \pm 8.81$ & $56.83 \pm 9.58$ & 0.305 & 0.822 \\
\hline
\end{tabular}

LA, luminal A; LB, luminal B; TNBC, triple negative breast cancer.

Table 3 Tumor size of each subtype of the breast cancer group

\begin{tabular}{lcccccc}
\hline Tumor size & LA & LB HER2 $(+)$ & HER2 $(+)$ & TNBC & $\chi^{2}$ & P value \\
\hline T1 & 10 & 12 & 12 & 16 & 14 & 0.773 \\
T2 & 19 & 16 & 15 & 282 & \\
T3 & 1 & 2 & 3 & 2 & \\
\hline
\end{tabular}

LA, luminal $A$; LB, luminal $B$; TNBC, triple negative breast cancer.

0.5020. Finally, 162 co-expressed genes of EGFR and 264 coexpressed genes of $E Z H 2$ were screened out. STRING (13) online tools were used to construct a protein-protein interaction (PPI) network, and Cytoscape (14) software was applied to screen out potential functional modules and to analyze the biological functions.

\section{Statistical analysis}

SPSS 25.0 software was used to perform the statistical analysis. The qRT-PCR mRNA expression data was expressed as $\bar{x} \pm$ s. Two groups of data were compared using the $t$-test, and multiple groups of data were compared using analysis of variance or the rank-sum test. Western blot results were analyzed using Image $\mathrm{J}$ software. The correlation between EGFR and EZH2 expression was analyzed by Pearson correlation analysis, and the correlation between coexpressed genes was analyzed by the Spearman correlation test. The strength of the correlation was determined by the following criteria: $0.00-0.19$ "very weak", $0.20-0.39$ "weak", 0.40-0.59 "medium", 0.60-0.79 "strong”, 0.80-1.0 "very strong". P value $<0.05$ was considered statistically significant.

\section{Results}

\section{Patient baseline data}

The comparisons between the breast cancer group and the benign breast lesions group were shown in Table 1. The differences between all subtypes of the breast cancer group were shown in Table 2. The tumor sizes of each subtype of the breast cancer group were shown in Table 3 .

\section{EGFR and EZH2 were bighly expressed in breast cancer tissues}

Western blot and qRT-PCR results showed that the protein and mRNA expression levels of EGFR and EZH2 in breast cancer tissues were higher than those in benign breast lesions (Tables 4,5).

\section{The expression levels of EGFR and EZH2 proteins in each subtype of the breast cancer group}

In the 120 cases of breast cancer tissues, 55 cases were positive for EGFR protein expression, and the western 
Table 4 Protein expression of EGFR and EZH2 in benign breast tissues and breast cancer

\begin{tabular}{ccccc}
\hline Gene & Benign & Breast cancer & $\chi^{2}$ & P value \\
\hline EGFR & & & 5.011 & 0.025 \\
+ & 7 & 55 & & \\
- & 23 & 65 & & \\
EZH2 & & & 12.069 & 0.001 \\
+ & 9 & 78 & & \\
- & 21 & 42 & & \\
\hline
\end{tabular}

$E G F R$, epidermal growth factor receptor; $E Z H 2$, zeste gene enhancer homolog 2.

Table 5 mRNA expression of EGFR and EZH2 in benign breast tissues and breast cancer

\begin{tabular}{lcccc}
\hline Gene & Benign & Breast cancer & $\mathrm{t}$ & $\mathrm{P}$ value \\
\hline EGFR & $0.493 \pm 0.294$ & $0.684 \pm 0.317$ & 3.105 & 0.026 \\
EZH2 & $0.328 \pm 0.144$ & $0.471 \pm 0.239$ & 2.134 & 0.040 \\
\hline
\end{tabular}

$E G F R$, epidermal growth factor receptor; $E Z H 2$, zeste gene enhancer homolog 2.

blot results are shown in Figure 1A. In the positive group, the expression levels of EGFR protein in luminal A (LA), luminal B (LB) HER2(+), HER2(+), and TNBC were $0.392 \pm 0.152,0.584 \pm 0.217,0.922 \pm 0.547$, and $1.474 \pm 0.556$, respectively (Figure 1B). The EGFR expression of TNBC was significantly different from the LA and LB HER2(+) types $(\mathrm{P}<0.05)$, while there was no significant difference among the other subtypes.

Among the 120 breast cancer tissues, 78 cases were positive for $E Z H 2$ protein expression. The western blot results are shown in Figure $2 A$. The expression levels of EZH2 protein in LA, LB HER2(+), HER2(+), and TNBC were $0.367 \pm 0.154,0.628 \pm 0.204,0.659 \pm 0.227$, and $1.079 \pm 0.330$, respectively (Figure $2 B$ ). The expression of $E Z H 2$ protein in TNBC was significantly different from other subtypes $(\mathrm{P}<0.05)$, and there was no significant difference between the remaining subtypes.

\section{Expression of EGFR and EZH2 mRNA in each subtype of breast cancer}

The expression levels of EGFR mRNA in LA, LB HER2(+), HER2(+), and TNBC were $0.429 \pm 0.350,0.602 \pm 0.373$,

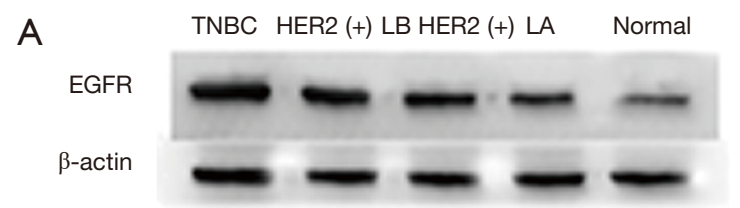

B

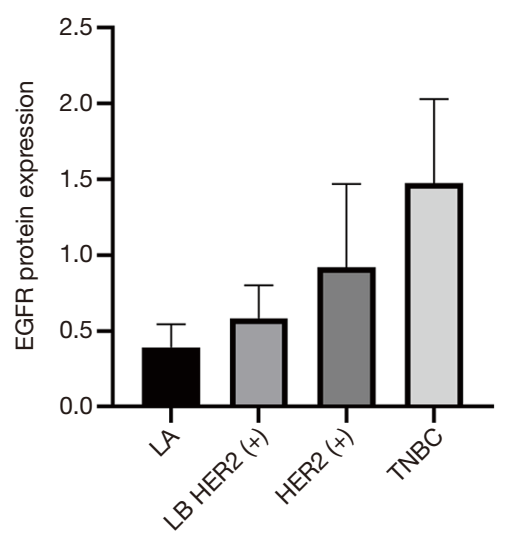

Figure 1 EGFR protein expression in different molecular subtypes of breast cancer. (A) Western blot results of each subtype of breast cancer. (B) EGFR protein expression of each subtype of breast cancer. EGFR, epidermal growth factor receptor; LA, luminal A; $\mathrm{LB}$, luminal B; TNBC, triple negative breast cancer.

$0.805 \pm 0.130$, and $0.902 \pm 0.098$, respectively (Figure $3 A$ ). EGFR expression in TNBC was significantly different from LA type $(\mathrm{P}<0.05)$, and the difference between HER2 $(+)$ and LA type was also statistically significant $(\mathrm{P}<0.05)$. However, there was no significant difference between the other subtypes.

The expression levels of EZH2 mRNA in LA, LB HER2(+), HER2(+), and TNBC were $0.235 \pm 0.115$, $0.375 \pm 0.246,0.559 \pm 0.251$, and $0.805 \pm 0.191$, respectively (Figure 3B). There were significant differences between TNBC, LA, and LB HER2(+) types. At the same time, there were significant differences between LA and LB HER2(+) types, but there was no significant difference between the remaining subtypes.

\section{Relationship between EGFR, EZH2, and clinicopathological factors}

We analyzed the correlations between patient clinical data and EGFR and EHZ2 expression. As shown in Table 6, the simultaneous high expression of both EGFR and EHZ2 mRNA was related to lymph node metastasis and clinical stage $(\mathrm{P}<0.05)$. 
A

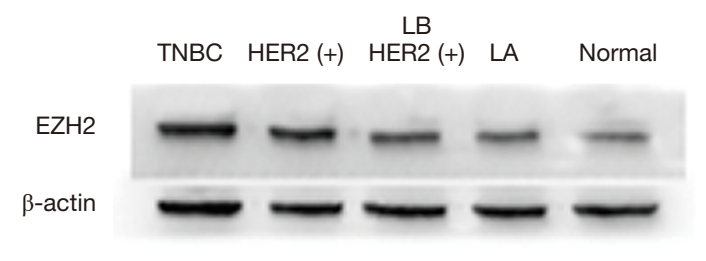

B

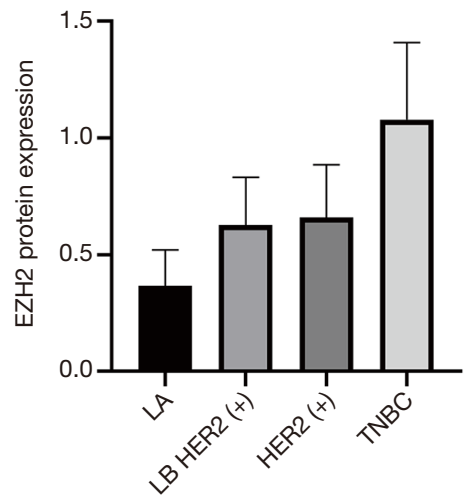

Figure 2 EZH2 protein expression in different subtypes of breast cancer. (A) Western blot results of each subtype of breast cancer. (B) EZH2 protein expression of each subtype of breast cancer. EZH2, zeste gene enhancer homolog 2; LA, luminal A; LB, luminal B; TNBC, triple negative breast cancer.

A

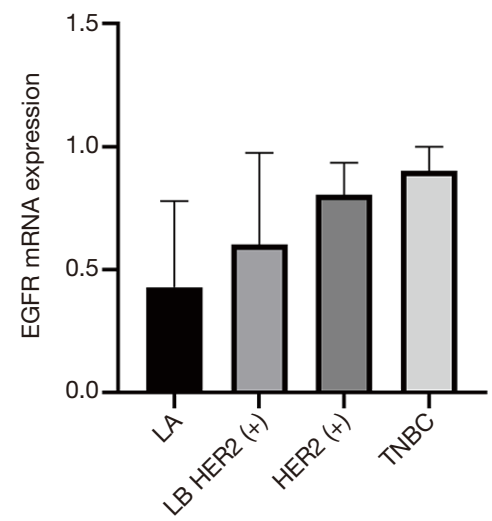

B

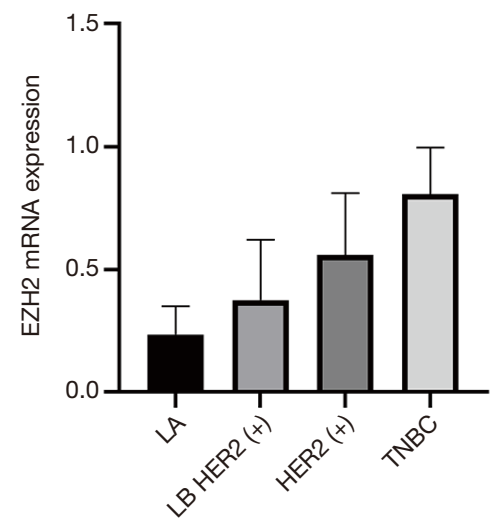

Figure 3 mRNA expression in different molecular subtypes of breast cancer. (A) EGFR mRNA expression of each subtype of breast cancer. (B) EZH2 mRNA expression of each subtype of breast cancer. EGFR, epidermal growth factor receptor; EZH2, zeste gene enhancer homolog 2; LA, luminal A; LB, luminal B; TNBC, triple negative breast cancer.

\section{Screening of co-expressed genes and establishment of the PPI network}

\section{PPI network analysis of EGFR}

STRING and Cytoscape software were used to establish a PPI network of co-expressed genes. The MCODE plug-in was used to filter out the main functional modules in the network (Figure 4A, indicated in yellow). Meanwhile, the cytoHubba plug-in was used to screen out the top 10 genes [top 10 nodes ranked by maximal clique centrality (MCC)] (Figure 4B). These genes were consistent with the enrichment of functional modules screened by MCODE.

The hub genes were enriched by Gene Ontology (GO) and Kyoto Encyclopedia of Genes and Genomes
(KEGG) pathway analysis through the STRING database. The results showed that these genes were involved in the biological processes of keratinization, epithelial development, epithelial cell differentiation, estrogen signaling pathways, breast cancer, prolactin signaling pathways, and acute granulocytes. The complete KEGG enrichment was shown in Table S1.

\section{PPI network analysis of EZH2}

STRING and Cytoscape software were used to establish the PPI network of co-expressed genes. The MCODE plug-in and cytoHubba plug-in were used to filter out the main functional modules in the network (Figure $5 \mathrm{~A}$, indicated in yellow) and the top 10 genes (top 10 nodes ranked by MCC) (Figure 5B), respectively. The results of 
Table 6 Relationship between EGFR and EZH2 expression and clinicopathological factors

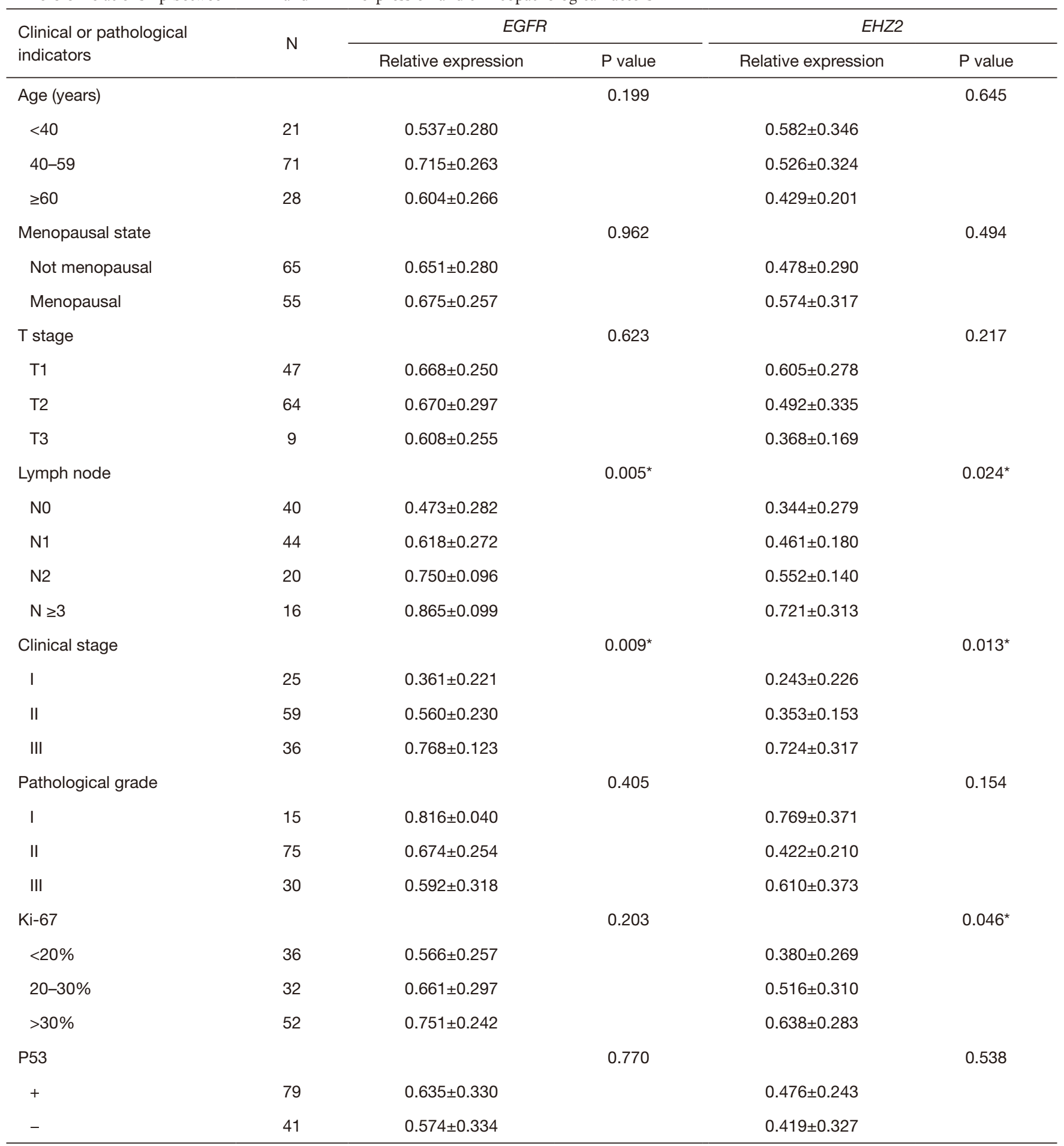

*, indicates the result is statistically significant. EGFR, epidermal growth factor receptor; $E Z H 2$, zeste gene enhancer homolog 2. 
A

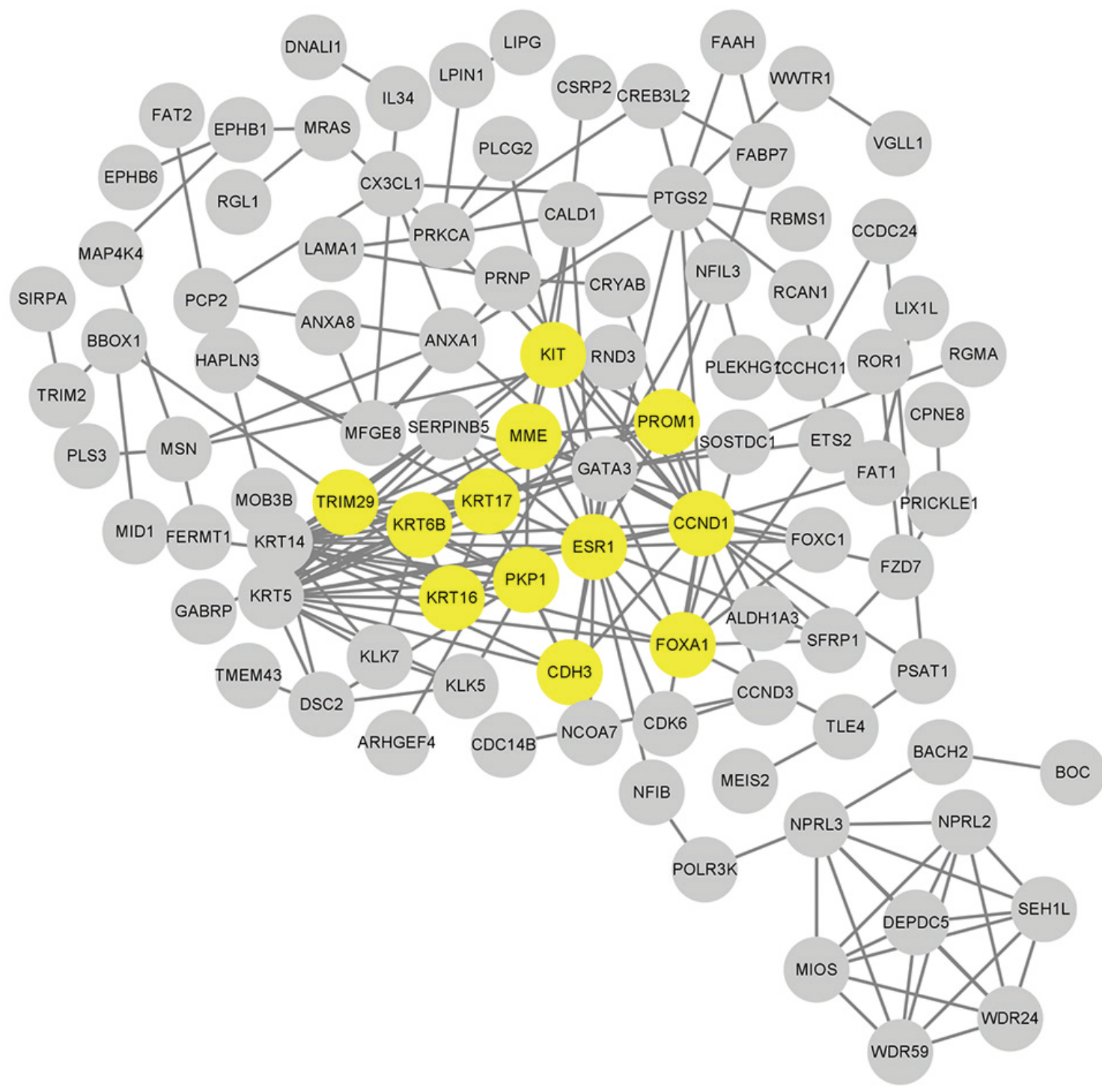

B

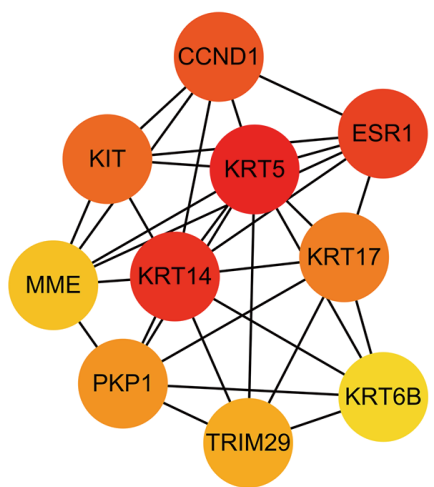

Figure 4 Co-expressed gene protein interaction network of EGFR. (A) The most important functional modules in the PPI network (indicated in yellow). (B) Hub genes screened by cytoHubba. EGFR, epidermal growth factor receptor; PPI, protein-protein interaction. 


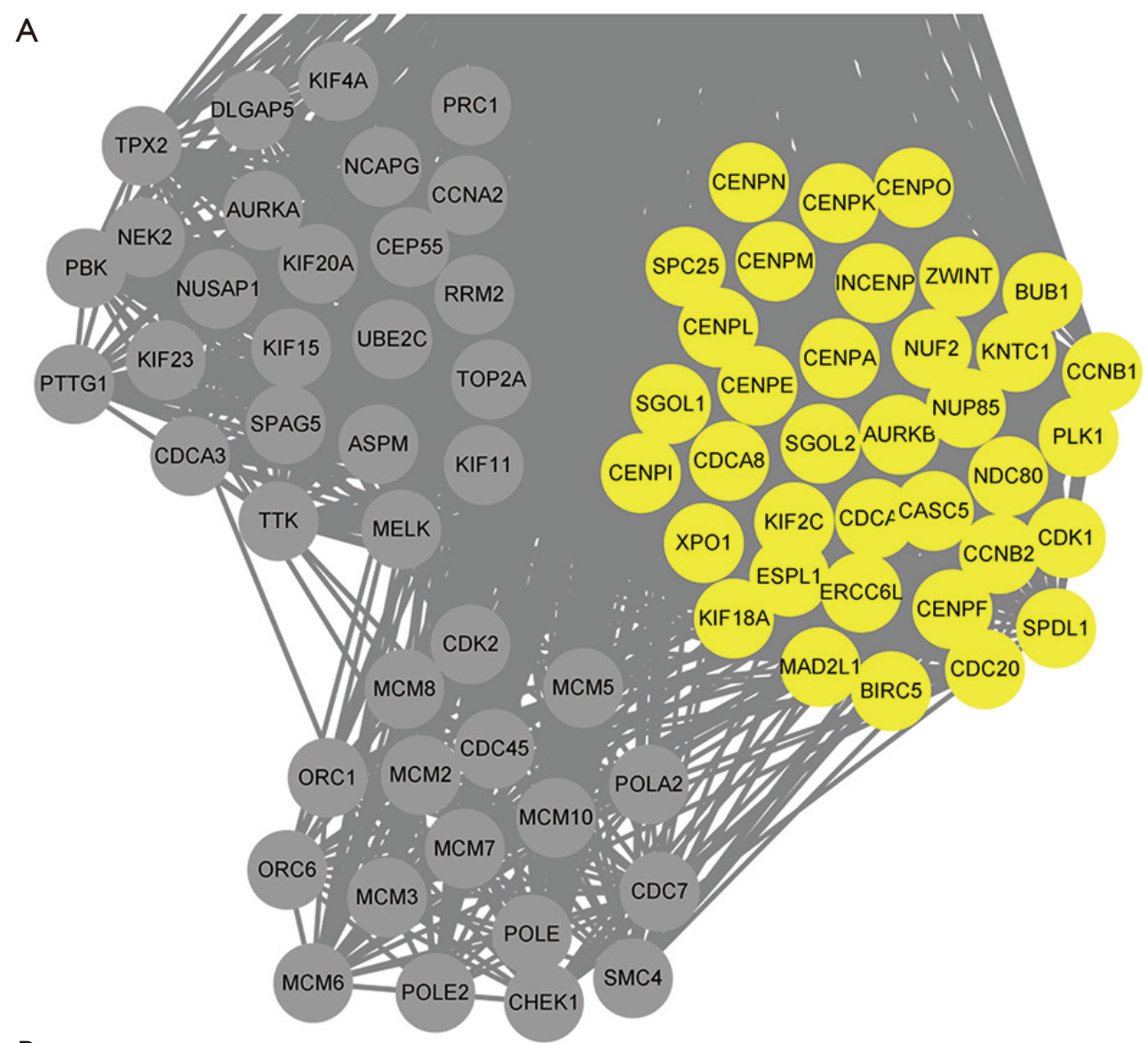

B

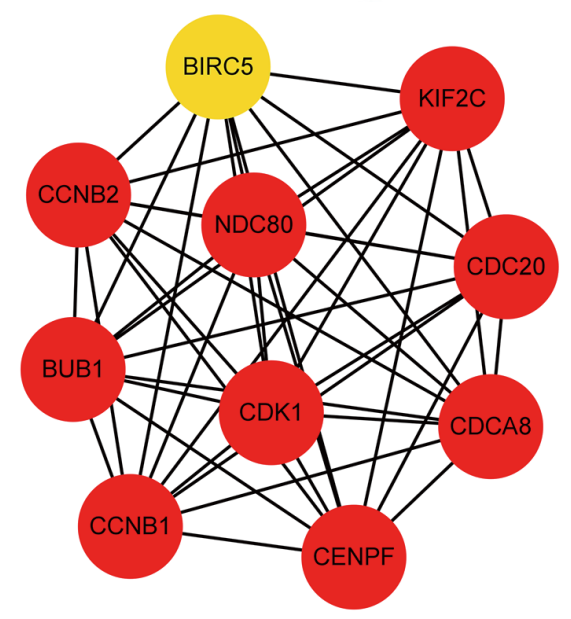

Figure 5 Co-expressed gene protein interaction network of EZH2. (A) The most important functional modules in the PPI network (indicated in yellow). (B) Hub genes screened by cytoHubba. EZH2, zeste gene enhancer homolog 2; PPI, protein-protein interaction.

the functional modules and the hub genes were consistent.

Enrichment analysis of hub genes using the STRING database showed that they were involved in cell division, mitotic cell cycle process, chromosome separation, chromosome location establishment, and other biological processes, as well as the cell cycle, p53 signaling pathway, cell senescence, FOXO signaling pathway, etc. The complete KEGG enrichment was shown in Table S2. 


\section{Discussion}

$E G F R$ is mainly converted into dimers by binding to ligands, and affects downstream pathways through autophosphorylation, thereby regulating cell functions. At present, there are two typical pathways known to be involved. One is the Ras-Raf-MAPK pathway, also known as the mitogen-activated protein kinase pathway, which regulates the cell cycle and cell proliferation-related functions through the activation of MAPK, ERK1, and ERK2 kinases. A research pointed out that it plays an important role in the metastasis of breast cancer cells (15). The other is the PI3K-AKT-mTOR pathway, which mainly regulates the anti-apoptotic-related responses of cells by activating serine protein kinase (16). This pathway is also a typical pathway involved in the development of HER2 overexpression and TNBC. Inhibition of this pathway can improve the prognosis of patients (17). In this study, the expression of EGFR in TNBC and HER2-overexpressed breast cancer was higher than that in LA breast cancer, which was consistent with the results of Su et al. (18). Hub gene enrichment analysis results showed that these genes were enriched in the estrogen signaling pathway, breast cancer, and prolactin signaling pathway, indicating that $E G F R$ may be involved in the negative transformation processes of TNBC-related receptor targets.

EZH2 mainly acts as a transcriptional inhibitor by catalyzing the trimethylation of lysine 27 (H3K27me3) of histone H3. The methylated $\mathrm{H} 3 \mathrm{~K} 27$ me3 recruits PRC2 complexes to specific gene sites which silences the expression of target genes and promotes tumor metastasis (19). A study observed that reducing the expression of EZH2 in the cytoplasm by inhibiting its upstream factors can inhibit tumor metastasis (20). The hub gene analysis of EZH2 demonstrated that the above genes were mainly enriched in cell cycle-related biological processes such as mitosis, nuclear division, and chromosome separation, indicating that EZH2 may be involved in the progression of breast cancer by affecting the proliferation potential and clonality of cancer cells. In this study, the expression of $E Z H 2$ protein in TNBC was significantly higher than in other subtypes. Nie et al. (21) found that the inhibition of EZH2 and cyclin-dependent kinase 2 (CKD2) could re-express the estrogen receptor of TNBC tumor cells, thereby enabling the use of tamoxifen for endocrine therapy, which suggests that EZH2 may also participate in the transformation process of TNBC.

The hub genes of EZH2 were enriched in the FOXO signaling pathway, and the FOXO signaling pathway is one of the downstream pathways in which MAPK and PI3K work together. It acts on a variety of biological metabolic processes and key cancer metabolic pathways to affect tumor cell metabolism (22). A study pointed out that the up-regulation of FOXO3A, a member of the FOXO family, promotes angiogenesis, thereby enhancing the metastatic invasiveness of breast cancer cells (23). The FOXO family also plays an important role in the metastasis of many cancers (24-26). One study noted that the activation or inhibition of FOXO inhibited the growth of metastatic tumor cells (27), which is different from the role of FOXO as a tumor suppressor gene in traditional cognition, indicating that a certain amount of intracellular expression of FOXO may be necessary to maintain tumor cell survival. In this study, the protein and mRNA expression of EGFR and $E Z H 2$ were positively correlated. It may be that the regulatory effects of EGFR and EZH2 converge in the downstream FOXO pathway. The high expression of the two in TNBC may be related to the high proliferation and metastasis of TNBC. However, only high-level mRNA expression of EGFR and EZH2 was related to prognostic factors such as lymph node metastasis and clinical staging $(\mathrm{P}<0.05)$. This indicates that other factors are involved in the regulation of the processes during protein translation, some scholars have reported examples of negative correlation between mRNA and protein expression (28). This study also has some limitations. There are not enough cases and few types, most of which are fibroadenoma of breast, the difference of mRNA expression in different types of benign breast lesions is difficult to be analyzed, large sample and further research are needed.

In short, by revealing the high expression of EGFR and $E Z H 2$ in breast cancer, especially TNBC, along with the correlation with clinicopathological factors and possible common signaling pathways, it is expected to become a potential therapeutic target. High mRNA expression was closely related to lymph node metastasis and clinical staging, making it an important molecular marker for the poor prognosis of breast cancer.

\section{Acknowledgments}

Funding: This work was supported by funding from the 2018 Guangxi Universities' Young and Middle-Aged Teachers' Basic Ability Improvement Project of Guangxi Education Department (2018KY0103). 


\section{Footnote}

Reporting Checklist: The authors have completed the REMARK reporting checklist. Available at https://dx.doi. org/10.21037/gs-21-505

Data Sharing Statement: Available at https://dx.doi. org/10.21037/gs-21-505

Conflicts of Interest: All authors have completed the ICMJE uniform disclosure form (available at https://dx.doi. org/10.21037/gs-21-505). The authors have no conflicts of interest to declare.

Ethical Statement: The authors are accountable for all aspects of the work in ensuring that questions related to the accuracy or integrity of any part of the work are appropriately investigated and resolved. All procedures performed in this study involving human participants were in accordance with the Declaration of Helsinki (as revised in 2013). The study was approved by institutional ethics board of First Affiliated Hospital of Guangxi Medical University [No. 2021(KY-E-038)] and informed consent was taken from all the patients.

Open Access Statement: This is an Open Access article distributed in accordance with the Creative Commons Attribution-NonCommercial-NoDerivs 4.0 International License (CC BY-NC-ND 4.0), which permits the noncommercial replication and distribution of the article with the strict proviso that no changes or edits are made and the original work is properly cited (including links to both the formal publication through the relevant DOI and the license). See: https://creativecommons.org/licenses/by-nc-nd/4.0/.

\section{References}

1. Bray F, Ferlay J, Soerjomataram I, et al. Global cancer statistics 2018: GLOBOCAN estimates of incidence and mortality worldwide for 36 cancers in 185 countries. CA Cancer J Clin 2018;68:394-424.

2. Li H, Zheng RS, Zhang SW, et al. Incidence and mortality of female breast cancer in China, 2014. Zhonghua Zhong Liu Za Zhi 2018;40:166-71.

3. $\mathrm{Hu} \mathrm{L}, \mathrm{Bu} Z$, Huang $\mathrm{G}$, et al. Assisted reproductive technology in china: results generated from data reporting system by CSRM from 2013 to 2016. Front Endocrinol (Lausanne) 2020;11:458.
4. Fan L, Strasser-Weippl K, Li JJ, et al. Breast cancer in China. Lancet Oncol 2014;15:e279-89.

5. Yarden Y, Pines G. The ERBB network: at last, cancer therapy meets systems biology. Nat Rev Cancer 2012;12:553-63.

6. Rose M, Maurer A, Wirtz J, et al. EGFR activity addiction facilitates anti-ERBB based combination treatment of squamous bladder cancer. Oncogene 2020;39:6856-70.

7. Rossini A, Giussani M, Ripamonti F, et al. Combined targeting of EGFR and HER2 against prostate cancer stem cells. Cancer Biol Ther 2020;21:463-75.

8. Talukdar S, Emdad L, Das SK, et al. EGFR: An essential receptor tyrosine kinase-regulator of cancer stem cells. Adv Cancer Res 2020;147:161-88.

9. Liang Y, Xu X, Wang T, et al. The EGFR/miR-3383p/EYA2 axis controls breast tumor growth and lung metastasis. Cell Death Dis 2017;8:e2928.

10. Chu W, Zhang X, Qi L, et al. The EZH2-PHACTR2AS1-ribosome axis induces genomic instability and promotes growth and metastasis in breast cancer. Cancer Res 2020;80:2737-50.

11. Yomtoubian S, Lee SB, Verma A, et al. Inhibition of EZH2 catalytic activity selectively targets a metastatic subpopulation in triple-negative breast cancer. Cell Rep 2020;30:755-70.e6.

12. Gao J, Aksoy BA, Dogrusoz U, et al. Integrative analysis of complex cancer genomics and clinical profiles using the cBioPortal. Sci Signal 2013;6:pl1.

13. Szklarczyk D, Gable AL, Lyon D, et al. STRING v11: protein-protein association networks with increased coverage, supporting functional discovery in genomewide experimental datasets. Nucleic Acids Res 2019;47:D607-13.

14. Shannon P, Markiel A, Ozier O, et al. Cytoscape: a software environment for integrated models of biomolecular interaction networks. Genome Res 2003;13:2498-504.

15. Tang T, Zhu Q, Li X, et al. Protease Nexin I is a feedback regulator of EGF/PKC/MAPK/EGR1 signaling in breast cancer cells metastasis and stemness. Cell Death Dis 2019;10:649.

16. Lien EC, Dibble CC, Toker A. PI3K signaling in cancer: beyond AKT. Curr Opin Cell Biol 2017;45:62-71.

17. Bosch A, Li Z, Bergamaschi A, et al. PI3K inhibition results in enhanced estrogen receptor function and dependence in hormone receptor-positive breast cancer. Sci Transl Med 2015;7:283ra51.

18. Su YC, Burnouf PA, Chuang KH, et al. Conditional 
internalization of PEGylated nanomedicines by PEG engagers for triple negative breast cancer therapy. Nat Commun 2017;8:15507.

19. Rybak AP, Bristow RG, Kapoor A. Prostate cancer stem cells: deciphering the origins and pathways involved in prostate tumorigenesis and aggression. Oncotarget 2015;6:1900-19.

20. Anwar T, Arellano-Garcia C, Ropa J, et al. p38-mediated phosphorylation at T367 induces EZH2 cytoplasmic localization to promote breast cancer metastasis. Nat Commun 2018;9:2801.

21. Nie L, Wei Y, Zhang F, et al. CDK2-mediated site-specific phosphorylation of EZH2 drives and maintains triplenegative breast cancer. Nat Commun 2019;10:5114.

22. Yadav RK, Chauhan AS, Zhuang L, et al. FoxO transcription factors in cancer metabolism. Semin Cancer Biol 2018;50:65-76.

23. Mishra R, Thorat D, Soundararajan G, et al. Semaphorin 3A upregulates FOXO 3a-dependent MelCAM expression

Cite this article as: Tang X, Zhou T, Shen J, Luo M, Yuan H, Pan D, Li F. The expression and potential mechanism of EGFR and EZH2 in breast cancer. Gland Surg 2021;10(8):2535-2545. doi: $10.21037 /$ gs-21-505 leading to attenuation of breast tumor growth and angiogenesis. Oncogene 2015;34:1584-95.

24. Hou T, Li Z, Zhao Y, et al. Mechanisms controlling the anti-neoplastic functions of FoxO proteins. Semin Cancer Biol 2018;50:101-14.

25. Jiramongkol Y, Lam EW. FOXO transcription factor family in cancer and metastasis. Cancer Metastasis Rev 2020;39:681-709.

26. Ma J, Matkar S, He X, et al. FOXO family in regulating cancer and metabolism. Semin Cancer Biol 2018;50:32-41.

27. Hornsveld M, Smits LMM, Meerlo M, et al. FOXO transcription factors both suppress and support breast cancer progression. Cancer Res 2018;78:2356-69.

28. Lin X, Chai G, Wu Y, et al. RNA m6A methylation regulates the epithelial mesenchymal transition of cancer cells and translation of Snail. Nat Commun 2019;10:2065.

(English Language Editor: C. Betlzar) 


\section{Supplementary}

Table S1 Enrichment analysis results of hub genes of EGFR

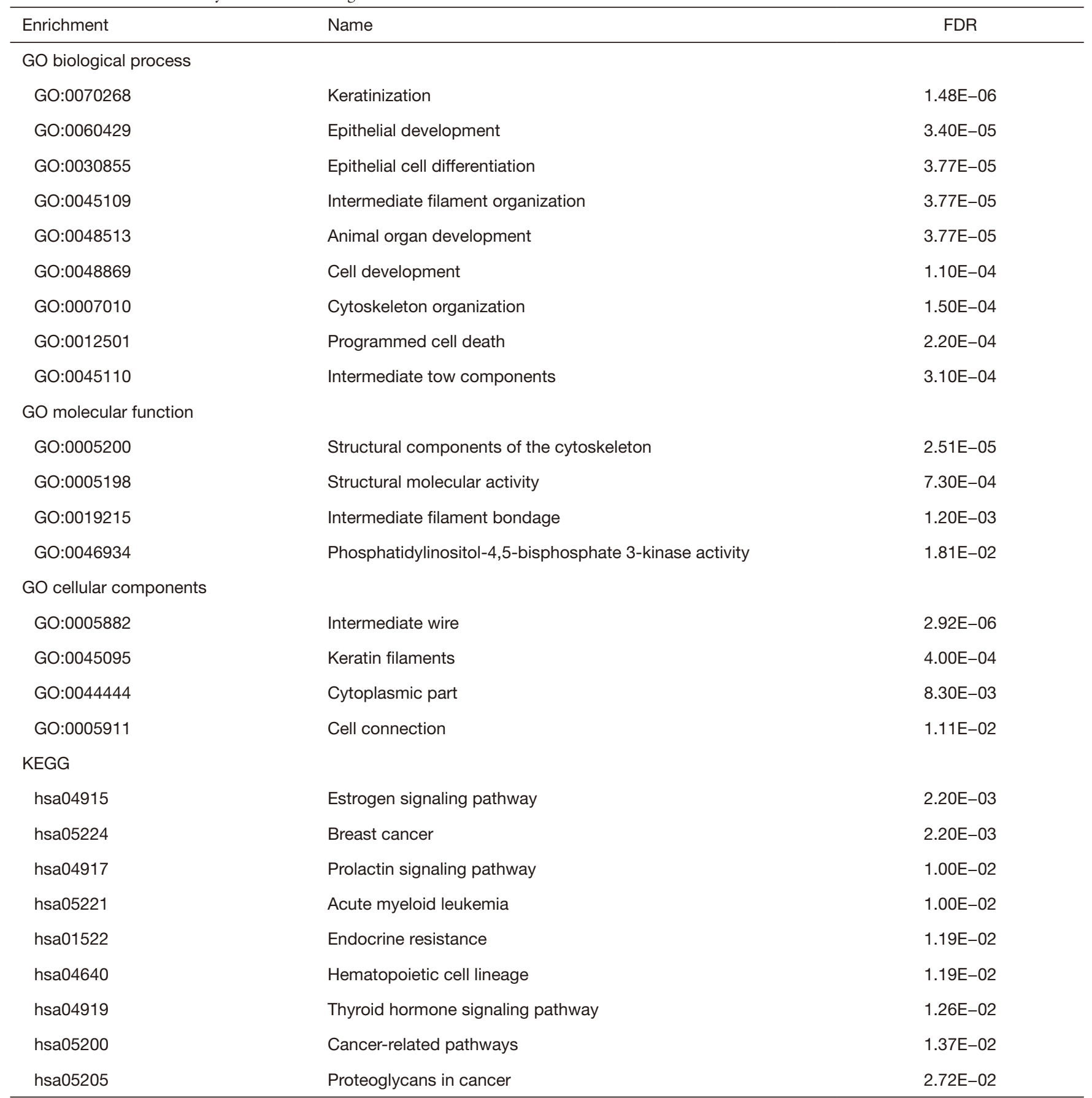

EGFR, epidermal growth factor receptor; GO, Gene Ontology; KEGG, Kyoto Encyclopedia of Genes and Genomes; FDR, false discovery rate. 
Table S2 Enrichment analysis results of hub genes of EZH2

\begin{tabular}{|c|c|c|}
\hline Enrichment & Name & FDR \\
\hline \multicolumn{3}{|c|}{ GO biological process } \\
\hline GO:0051301 & Cell division & $6.89 \mathrm{E}-14$ \\
\hline GO:1903047 & Mitotic cell cycle process & $1.60 \mathrm{E}-13$ \\
\hline GO:0007059 & Chromosome segregation & $7.24 \mathrm{E}-12$ \\
\hline GO:0051303 & Establishment of chromosome location & $8.34 \mathrm{E}-11$ \\
\hline GO:0098813 & Nuclear chromosome segregation & $1.20 \mathrm{E}-10$ \\
\hline GO:0051983 & Regulation of chromosome segregation & 2.77E-10 \\
\hline GO:0000280 & Nuclear division & $7.73 \mathrm{E}-10$ \\
\hline GO:0000819 & Sister chromatid separation & $9.15 \mathrm{E}-10$ \\
\hline GO:0140014 & Mitotic nuclear division & $1.41 \mathrm{E}-09$ \\
\hline GO:0030071 & Regulation of metaphase/late mitotic transition & $1.61 \mathrm{E}-09$ \\
\hline \multicolumn{3}{|c|}{ GO molecular function } \\
\hline GO:0004693 & Cyclin-dependent protein serine/threonine kinase activity & $7.20 \mathrm{E}-05$ \\
\hline GO:0035173 & Histone kinase activity & $1.30 \mathrm{E}-03$ \\
\hline GO:0004674 & Protein serine/threonine kinase activity & $1.40 \mathrm{E}-03$ \\
\hline GO:0140096 & Catalytic activity acting on protein & $3.13 E-02$ \\
\hline GO:0016740 & Transferase activity & $3.27 \mathrm{E}-02$ \\
\hline GO:0008022 & Protein C-terminal binding & $4.10 \mathrm{E}-02$ \\
\hline \multicolumn{3}{|c|}{ GO cellular components } \\
\hline GO:0000775 & Chromosome centromeric region & $1.14 \mathrm{E}-10$ \\
\hline GO:0000777 & Condensed chromosome kinetochore & $3.05 \mathrm{E}-10$ \\
\hline GO:0000940 & Condensed extrachromosomal kinetochore & $8.41 \mathrm{E}-10$ \\
\hline GO:0015630 & Microtubule cytoskeleton & $9.86 \mathrm{E}-10$ \\
\hline GO:0044430 & Cytoskeleton & $1.38 \mathrm{E}-08$ \\
\hline GO:0000942 & Condensed nuclear extrachromosomal kinetochore & $3.62 \mathrm{E}-08$ \\
\hline GO:0005819 & Spindle & $4.12 \mathrm{E}-08$ \\
\hline GO:0005815 & Microtubule Organization Center & $6.46 \mathrm{E}-08$ \\
\hline GO:0005813 & Centrosome & $2.72 \mathrm{E}-07$ \\
\hline GO:0043232 & Intracellular non-membrane organelles & $7.83 \mathrm{E}-07$ \\
\hline \multicolumn{3}{|l|}{ KEGG } \\
\hline hsa04110 & Cell cycle & $2.45 \mathrm{E}-08$ \\
\hline hsa04114 & Oocyte meiosis & $2.45 \mathrm{E}-08$ \\
\hline hsa04914 & Progesterone-mediated oocyte maturation & $4.85 \mathrm{E}-07$ \\
\hline hsa04115 & p53 signaling pathway & $1.62 \mathrm{E}-05$ \\
\hline hsa04218 & Cell senescence & $1.50 \mathrm{E}-04$ \\
\hline hsa04068 & FOXO signaling pathway & $3.90 \mathrm{E}-03$ \\
\hline hsa05203 & Viruses cause cancer & $6.50 \mathrm{E}-03$ \\
\hline hsa05166 & HTLV-I infection & 1.04E-02 \\
\hline
\end{tabular}

EZH2, zeste gene enhancer homolog 2; GO, Gene Ontology; KEGG, Kyoto Encyclopedia of Genes and Genomes; FDR, false discovery rate. 